\title{
Managing uncertainty in decision support models foreword to the special issue
}

In September 22-24, 2004, we served as chairs of the Organizing Committees and the International Programme Committee of the 15th Mini-EURO Conference on Managing Uncertainty in Decision Support Models (MUDSM 2004) held at the University of Coimbra, Portugal. The scientific program included 20 sessions where 63 papers were presented, and 78 researchers from 24 countries attended this event.

Mini EURO Conferences are specialized events, under the auspices of EURO-The Association of European Operational Research Societies, allowing researchers and practitioners in specific Operational Research (OR) areas to participate in meetings where the screening of papers can be more effective and participation in the debates can be more lively than at larger and with broader scope conferences. The 15th Mini EURO Conference was aimed at providing an open forum in which researchers and practitioners coming from different scientific disciplines and OR areas could discuss and share their experience regarding methodological approaches to tackle uncertainty stemming from distinct sources, which have influence on obtaining robust conclusions in decision support models, with application to several areas. Types and sources of uncertainties such as the intrinsic incomplete nature of models, the imprecision associated with input data, the analytical representation of the structure of preferences of the Human decision maker, etc., were addressed by the MUDSM 2004 participants. Contributions from different methodological areas (such as decision theory, fuzzy sets, rough sets, risk analysis, stochastic programming, sensitivity analysis, interval programming, etc.) were presented and applications in different relevant fields were described, for tackling uncertainty, imprecision and risk in decision support models.
In modern complex technological societies uncertainty is inherent to almost all decision problems. From lack or imperfection of knowledge to structural uncertainty, such as the adaptive behavior of humans, including the scientists' choices of models and methods, uncertainty is pervasive in most decision contexts. In such turbulent environment, scientists are often pressed to generate recommendations with degrees of certainty that cannot be sustained. However, rigor and intellectual honesty impose that scientists must explicitly incorporate into their models and studies the intrinsic uncertainty arising in complex problems, namely decision problems in which multiple axes of evaluation are at stake. In almost all fields, namely in applied ones, the imprecise, the uncertain, the unknown are innate components of problems to be tackled. Therefore, managing uncertainty in decision support models explicitly addresses the sources and types of uncertainty arising in decision problems and provides methodological tools and adaptive frameworks aimed at helping to reach robust decisions in decision support processes. Although this often leads to models that are more mathematically sophisticated, the use of computerbased decision support systems contributes to facilitate the decision-makers' tasks in real-world problems.

The authors of papers more related to the use of computer-based decision aids were invited to submit extended versions of the papers presented at the conference to this special issue. Eight papers were selected after a thorough review supervised by the guest editors (with the exception of the paper by Damart et al., for which this process was supervised by C.H. Antunes alone). The papers are herein presented by alphabetical order.

Brynielsson proposes a decision support tool for Command and Control situation awareness enhancements 
based on game theory for inference, which is coupled with traditional AI methods for uncertainty modeling. This approach is aimed at capturing situations where commanders make decisions based on other commanders' reasoning about one's own reasoning (that is, where the optimization of decisions in complex multi-agent environments is at stake), which is not generally possible using traditional agent modeling techniques. The game-theoretic analysis techniques are illustrated using with a realworld examples on the tactical level.

Damart, Dias and Mousseau address the situation in which a group interacts to cooperatively develop a common multicriteria evaluation model to sort actions into classes. A methodology is proposed in which the group discusses the assignment of some exemplary actions (possibly fictitious) to categories as a means to infer a sorting model, instead of specifying values for the model parameters. The collective model is (imprecisely) defined by the consistent examples agreed by the group. The sorting examples relate to constraints on the parameter values, which is a case of partial information. The methodology is based on an aggregation/disaggregation approach for the ELECTRE TRI method, implemented on the Decision Support System IRIS, which is suited to contexts where numerical information about preferences is hard to obtain.

French explores the implication of results from voting theory and social choice to suggest that Group DSS and web-enabled GDSS require much more sensitive, less algorithmic approaches to decision support than DSS built for individual decision makers. The discussion suggests that web-enabled GDSS needs to address problems of communication and explanation much more than algorithmic details of voting rules. The author defends that failure of GDSS designers to address the implications of some known results, such as the ones from voting theory and social choice, may lead to the development of inappropriate tools. This means that valid web-enabled group decision support and edemocracy need address communication and explanation within the human-computer interface in a consistent manner.

Jiménez, Mateos, Ríos-Insua and Rodríguez present a decision analysis methodology, and a decision support system to allay the operational difficulties associated with the selection of a supplier for cleaning services in a European public underground transportation company, according to the European Community directives. The model explicitly considers multiple and conflicting criteria, such as improving service levels and reducing total service costs. This DSS is able to tackle incomplete information about the decision-maker's preferences, also accounting for the uncertainty associated with offer performances (input imprecision).

Recognizing the growing interest of citizens to participate in local policy making processes that affect their daily life, Lourenço and Costa propose a system for supporting public participation using information and decision technologies (e-participation), which is based on a discussion structure template to organize the contributions and employing collaborative writing processes to generate agreed documents. These documents may then be used as formal input into the policy making process thus incorporating the citizens' views on those issues. Public participation is herein seen as a way to reduce the uncertainty, particularly with respect to the consequences of feasible actions, and the complexity associated with local policy issues and to improve the democratic legitimacy of those processes.

Ozturk and Tsoukiàs explore the interpretation of a continuous extension of a four-valued logic as a necessity degree (in possibility theory), for modeling uncertain positive and negative reasons in decision aiding. The authors claim that the design and implementation of Decision Support Systems requires the use of formal languages to code the information about decision problems and decision makers' preferences, but classical logic is not always adequate to formalize situations in which it is necessary to handle incomplete and/or inconsistent information. The paper aims at verifying whether it is possible to associate to the DDT logic an uncertainty distribution. DDT logic is a fourvalued logic to model continuous valuations on the presence of truth extended as a language for preference modeling purposes.

Petrovic, Duenas, and Petrovic present a decision support tool to deal with multi-objective job shop scheduling problems, which includes an interactive fuzzy multi-objective genetic algorithm (GA). The fitness function is defined as a measure of truth of a linguistically quantified statement, imprecisely specified by the decision maker using linguistic quantifiers (modeled using fuzzy sets), with respect to acceptable deviations between the achieved objective values and the aspiration levels. A real-world problem in the pottery industry is described.

Salling, Leleur and Jensen describe a decision support system, which enables to assess various uncertainties in project appraisal, in the framework of a large infrastructure project - the Øresund fixed link in Denmark. This DSS has been developed within the Danish Centre for Logistics and Freight Transport, for 
an ex-post appraisal study, and it is based on costbenefit analysis embedded in multi-criteria analysis (MCA). A general equilibrium model is applied to calibrate the MCA component. The model also makes use of the link between scenario analysis (SA) and Monte Carlo simulation (MCS), handling uncertain information across the assessment criteria and the application of SA to inform the MCS parameter setting.

We would like the Organizing Committee and the International Programme Committee, which we had the honor to chair, for their efforts which greatly contributed to the success of MUDSM 2004, the Editor of Decision Support Systems journal, as well as the contributors and all the reviewers involved in assessing the merits of the contributions submitted for publication in this feature issue.
Carlos Henggeler Antunes Department of Electrical Engineering and Computers, University of Coimbra, Polo II, 3030-290 Coimbra, Portugal INESC Coimbra, Rua Antero de Quental, 3000-033 Coimbra, Portugal E-mail address: cantunes@inescc.pt.

Luís Cândido Dias Faculty of Economics, University of Coimbra, Av. Dias da Silva 165, 3004-512 Coimbra, Portugal INESC Coimbra, Rua Antero de Quental, 3000-033 Coimbra, Portugal E-mail address: 1dias@inescc.pt. Corresponding author. 